\title{
Hackathon aims to solve materials problems
}

$\mathrm{B}$ leary eyes and a taxed brain are common at Materials Research Society (MRS) Meetings, but for the first time, a group of intrepid attendees had a very different reason for their weariness than the typical gauntlet of talks and networking sessions. During the 2014 MRS Fall Meeting in Boston, 14 materials scientists came together for 24 hours for MatHack, the world's first materials hackathon, to solve real materials problems. A hackathon is a sprint computer programming competition where participants collaborate to create software from scratch in intense sessions over one or two days. Sponsored, in part, by Citrine Informatics and driven by two of its founders (Bryce Meredig and Greg Mulholland) in collaboration with the MRS Academic Affairs Committee, MatHack participants pitched ideas, formed teams, spent one night writing code, and presented their work to a panel of judges from across the materials community.
The idea behind a hackathon is to very quickly build functional (yet imperfect) software to lay the foundation for further development in the future. Such events are common in Silicon Valley; Google and Facebook are renowned hackathon hosts and sponsors. Mark Zuckerberg, founder and chief executive officer of Facebook, has explained that "hacking just means building something quickly or testing the boundaries of what can be done."1 The participants at the first MatHack did just that, demonstrating that even in a field like materials science - traditionally associated with longer-term laboratory investigations - people can produce creative, meaningful scientific software contributions in a very short time.

MatHack participants hailed from wideranging backgrounds within the materials community. Some were experienced computational materials scientists with tremendous coding backgrounds. Others were experimentalists with practical problems

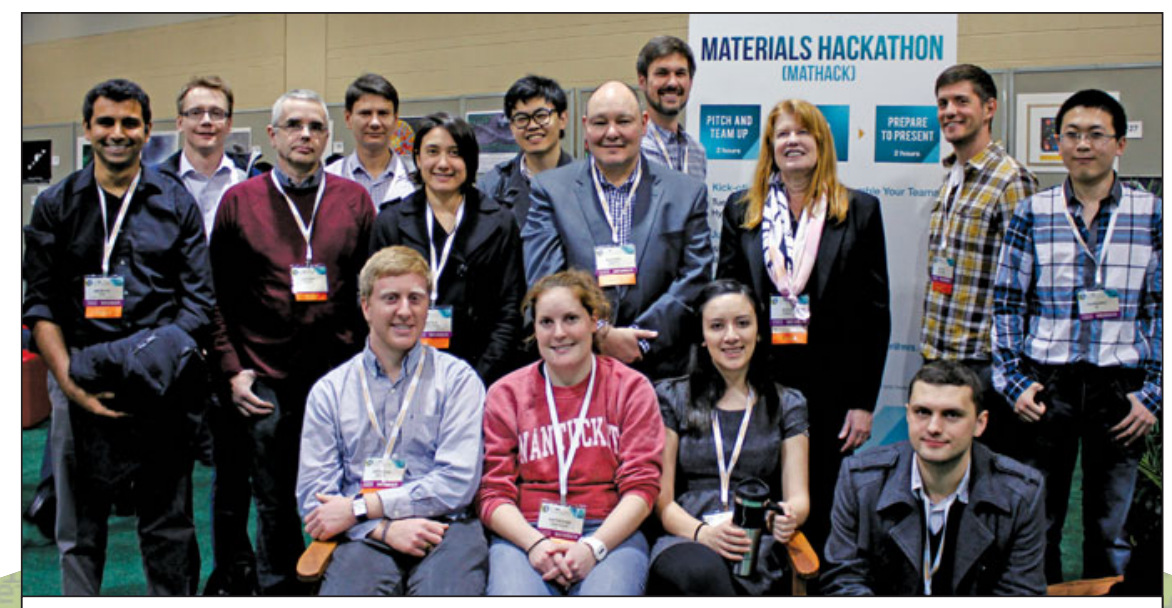

Hackathon participants, judges, and organizers (front row, left to right): Brendan Nagle (Dartmouth College), Katie Van Aken (Drexel University), Sabrina Ball (MIT), loan-Bogdan Magdau (The University of Edinburgh); (second row, left to right): Anubhav Jain (Lawrence Berkeley Lab), Timothy Large (Microsoft Applied Sciences), Nicole Adelstein (Lawrence Livermore National Lab), Rick Barto (Lockheed Martin), Susan Ermer (Lockheed Martin), Bryce Meredig (Citrine Informatics), Guoqiang Xu (MIT); (third row, left to right): Andre Schleife (Univ. Illinois at Urbana-Champaign), Oleg Rubel (McMaster/TBRRI), Wenhao Sun (MIT), Greg Mulholland (Citrine Informatics).

to solve as well as professors and postdocs with a desire to build something new. The participants included people from universities all over the world, spanning academia, industry, and national labs.

Participants first gathered in The Hub, the central area of the Fall Meeting, and gave 30 -second pitches for ideas that addressed a very broad prompt: "Build a piece of software that would be useful to a materials scientist." Some pitches were precise answers to lab pains that had simmered for years, while others were more general, outlining a rough idea of what a team might build together. While some projects could expand into entire doctoral dissertation topics, others tried to solve everyday problems that present themselves when working with materials, or proposed new means of outreach to inspire the next generation of diverse, engaged materials scientists. Ideas ranged from a tool to communicate the status of characterization tools within a lab, to automatically solving complex equivalent circuits, to density functional theory (DFT) approaches to quasicrystals, to crystal visualization.

After the pitches were complete, each presenter set up at a table, and for the next 30 minutes, participants met and talked with one another to learn more about the vision, technical challenges, and people involved with each idea. Then the teams were formed around many of these ideas. Most participants had never met before and built relationships over the course of the 24 hours.

Once teams formed, the real work began. While everyone had selected hard problems to solve, they did not all have the programming expertise or requisite knowledge to build the right systems. In some cases, people had an idea of how to execute from the outset; in other cases, they had to learn an entirely new programming language. The common thread, though, was a 
willingness and desire to learn new things, ask for help, and share ideas liberally. In typical hackathon fashion, sleep was scarce. Gallons of coffee and ice cream and pounds of nuts and other snacks fueled the teams through the night.

The next morning, participants presented their ideas to the panel of judges. This diverse group of judges then selected the top three teams for cash prizes. Though most of the faces were tired, no one appeared ready to stop working. Bruce Clemens, Stanford professor and former MRS President, said it best when he declared, "These teams blew me away!"

Hackathons often have themes, ranging from health care to civic engagement to education. MatHack was the first time that a hackathon's theme was materials science. Some projects will likely continue; others may not. What will certainly grow is the influence of software and hackathon thinking in materials at large. What can we accomplish if we boil materials challenges down to their bare essence and ask smart teams to focus intensely on them?

The collaborations and relationships that formed at MatHack, over a long night bathed in the glow of laptop screens, will certainly continue throughout the participants' scientific careers. This is the reason the MRS Meetings exist — to build such communities - and MatHack fits right into this mold.

\section{WINNERS}

\section{First Place: Phone-on Flow}

Nicole Adelstein and Andre Schleife

Team Phone-on Flow built a functioning three-dimensional crystal viewer. Using an Android phone, a student could visit a special web page and (using Google
Cardboard, ${ }^{2}$ take an immersive virtual tour through a crystal structure. Even to the judging panel, who has seen their share of crystals, the visualization was inspiring. As an outreach tool, such an inexpensive and interactive project could open up materials to a new generation of students.

\section{Second Place: MatHack QuasiCrystal Wenhao Sun}

Sun developed a new way to use DFT to simulate quasicrystals, which can be thought of as the material equivalent of the number $\pi$ : perfectly ordered but eschewing a repeating pattern. More importantly, they are a class of materials that shows promise for many applications but resists simple treatment with atomistic simulations. MatHack QuasiCrystal is the first step in being able to simulate these materials to understand them better.

\section{Third Place: Directed Materials Design (DMD)}

Andrew Long and Ioan-Bogdan Magdau Team DMD took an operations research approach to understanding materials. By looking at large-scale data sets, they built machine learning models to rapidly predict how various perovskite materials would work as water-splitting materials, without requiring expensive supercomputing time or the upfront investment of experiments.

\section{TEAMS}

\section{CIVR}

Sabrina Ball, Brendan Nagle, and Katie Van Aken

Team CIVR started with a clear question: "How can I automate the solving of equivalent RC circuits for electrochemical systems?" For the experimentalist, this is a hard problem. It involves solving huge sets of equations and fitting them to experimentally measured $I-V$ curves.

\section{MacHack}

Guoqiang Xu

MacHack is a tool to analyze the grain structure of a material using optical microscope images. It has a different take from most approaches, because it uses multiple images of the same spot under different lighting conditions, combined with machine learning to identify grain boundaries.

\section{MatMod}

\section{Gabriela Correa and Oleg Rubel}

Team MatMod wanted to address the problem of DFT (see text earlier) being computationally expensive. This is no small feat-Walter Kohn and John Pople shared the Nobel Prize in Chemistry in 1998 for developing the method and operationalizing it in working software. MatMod's approach was to use alternative $k$-point sampling techniques to reduce the complexity of DFT calculations for a particular system without substantially reducing accuracy.

\section{Greg Mulholland Bryce Meredig}

1. http://www.wired.com/2012/02/zuck-letter.

2. Google Cardboard is a fold-out cardboard smartphone mount that, when combined with lenses, a magnet, a piece of hook-and-loop fastener and a rubber band and held against the face, affords a virtual reality experience.
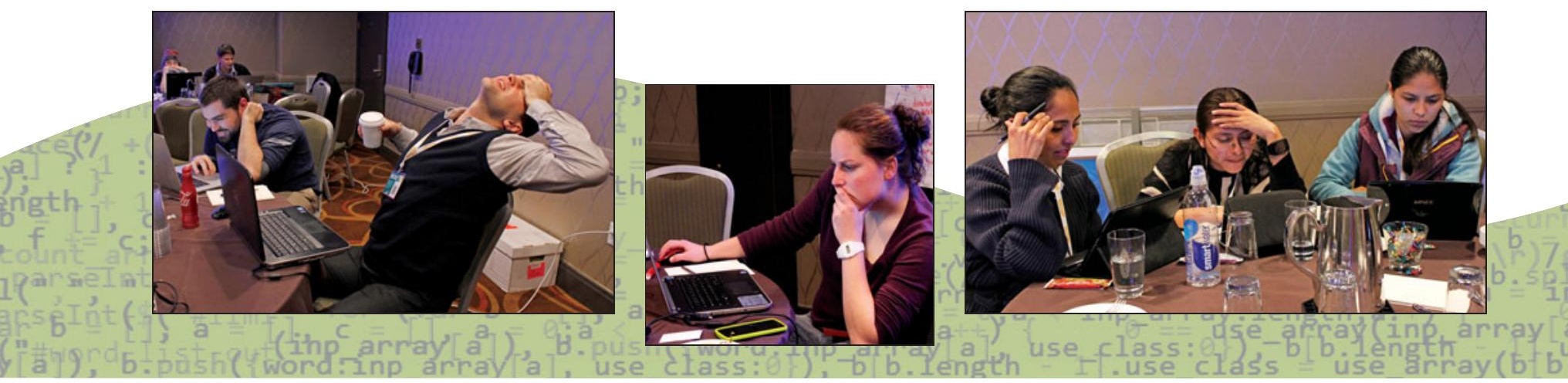


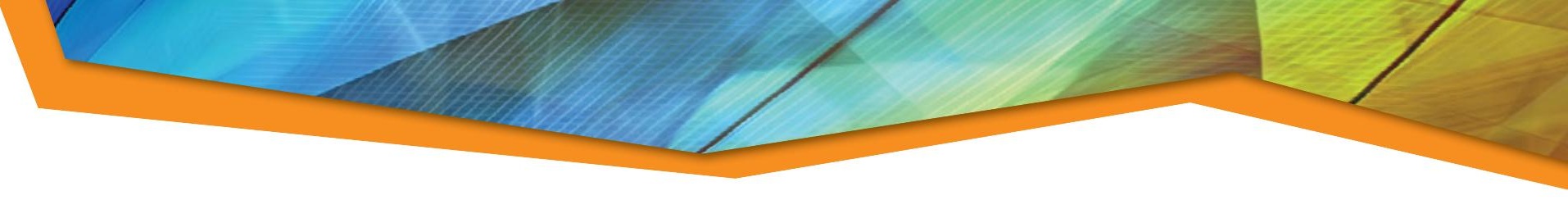

\section{Nanoindentation as a tool to measure and map mechanical properties of hardened cement pastes}

Chuanlin Hu, The Hong Kong University of Science and Technology, China

Regarding the significance of cement paste in construction materials, the present paper aims to use nanoindentation to measure and map mechanical properties of hardened cement pastes. The mechanical properties of involved phases were extracted from grid nanoindentation on the cement paste. The results suggested that nanoindentation can be used as a tool to measure and map mechanical properties of hardened cement pastes, and can identify the phases including outer product, inner product, calcium hydroxide (or interface of residual cement clinker), and residual cement clinker. DOI:10.1557/mrc.2015.3

\section{Novel one-pot synthesis of hierarchically porous $\mathrm{Pd} / \mathrm{C}$ monoliths by a co-gelation method}

Trupti V. Kotbagi, Yasemin Hakat, and Martin G. Bakker, The University of Alabama, USA

Described is a simple one-pot route for the synthesis of hierarchically porous carbon supported palladium $(\mathrm{Pd} / \mathrm{C})$ monoliths with a $3 \mathrm{D}$ pore network via self-assembly under basic conditions of a resol polymer. Textural and morphological characterization of the $\mathrm{Pd} / \mathrm{C}$ monoliths was carried out using nitrogen sorption analysis and SEM. Formation of Pd nanoparticles ranging from 35-60 nm was observed. Evaluation of catalytic activity for styrene hydrogenation was performed using the $\mathrm{Pd} / \mathrm{C}$ material as heterogeneous catalyst in batch mode. Studies revealed that the monoliths showed leaching and catalytic activity similar to a commercial $\mathrm{Pd} / \mathrm{C}$ catalyst. DOI:10.1557/mrc.2015.7

\section{Unique mechanical properties of fullerite derivatives synthesized with a catalytic polymerization reaction}

M. Popov and V. Blank, Technological Institute for Superhard and Novel Carbon Materials, National University of Science and Technology, and Moscow Institute of Physics and Technology State University, Russian Federation; S. Perfilov and

D. Ovsyannikov, Technological Institute for Superhard and Novel Carbon Materials, Russian Federation; B. Kulnitskiy, E. Tyukalova, V. Prokhorov, I. Maslenikov, Technological Institute for Superhard and Novel Carbon Materials, and Moscow Institute of Physics and Technology State University, Russian Federation; I. Perezhogin, Technological Institute for Superhard and Novel Carbon Materials, and Lomonosov Moscow State University, Russian Federation; E. Skryleva and Yu Parkhomenko, National University of Science and Technology, Russian Federation

Fullerite derivatives synthesized with a catalytic polymerization reaction at a relatively low-pressure range of $0.5-4 \mathrm{GPa}$ show unique mechanical properties: elastic recovery is $98 \%$ and hardness possibly approaches $100 \mathrm{GPa}$. Structure of the samples is also unique: one composes from fragments of $\mathrm{C}_{60}$ molecules linked by covalent bonds. To obtain the homogeneous crack-free samples, we synthesized a $\mathrm{B}_{4} \mathrm{C}$ - fullerite derivatives composite which show a $550 \mathrm{MPa}$ flexural stress, a $2250 \mathrm{MPa}$ compressive strength, and a $28 \mathrm{GPa}$ hardness and have density of $2.2 \mathrm{~g} / \mathrm{cm}^{3}$. DOI:10.1557/mrc.2015.4

\section{Ecofriendly synthesis of ultra small metal-doped $\mathrm{SnO}_{2}$ quantum dots}

Antonio Tirado-Guizar and Georgina Esther Pina-Luis, Instituto Tecnologico de Tijuana, México; Francisco Paraguay-Delgado, Centro de Investigación en Materiales Avanzados S.C., México

An ecofriendly synthesis is established to obtain ultra small $\mathrm{SnO}_{2}$ nanoparticles (NPs) doped with metals by a hydrothermal method using only tin tetrachloride, urea and water as reagents. This synthesis was done in a short period time at low temperature and without surfactants. Microscopy analysis revealed the formation of doped tin oxide NPs with a diameter smaller than $2.8 \mathrm{~nm}$. Un-doped and doped tin oxides were obtained with a tetragonal type rutile structure with an average surface area of $348 \mathrm{~m}^{2 /} \mathrm{g}$. DOI:10.1557/ mrc.2015.11

\section{Composing molecular music with carbon}

Ashley J. Kocsis and Steven W. Cranford, Northeastern University, USA

What musical notes can a molecule play? Carbyne is a chain of atoms that vibrates similar to an elastic string. Like the tuning of a guitar string, this vibration can be predicted based on length and tension. Using atomistic simulation, we determine the vibrational response of carbyne. We further produce audible notes, enabling specific musical composition with prescribed molecular conditions (pre-strain and length) and combine single chains into multichain systems to form molecular chords. Since the tension of a molecular chain is relatively low $(<\mathrm{nN})$, such "strings" can potentially be developed for signaling and detection with high resolution. DOI:10.1557/mrc.2015.9

\section{Indentation-induced two-way shape-memory effect in aged Ti-50.9 at.\% Ni}

Mareike Frensemeier and Eduard Arzt, INM-Leibniz Institute for New Materials, Saarland University, Germany; Enwei Qin, Suzhou Nuclear Power Research Institute Co., Ltd, China;

Carl P. Frick, University of Wyoming, USA; Andreas S. Schneider, INM-Leibniz Institute for New Materials, Germany

In this study, Vickers indentation was used to investigate the two-way shape memory effect (TWSME) in an austenitic Ti-50.9 at. \% Ni alloy, exposed to different heat treatments. Three aging treatments were used to manipulate the size of $\mathrm{Ti}_{3} \mathrm{Ni}_{4}$ precipitates. All samples were Vickers indented, and the indent depth was investigated as a functon of thermal cycling. The TWSME was found only in the material aged at $400^{\circ} \mathrm{C}$, which contained coherent precipitates. Thermal cycling shows stable TWSME, however, heating well above the austenite finish temperature lead to permanent austenitic protrusions. The results indicate that stabilized martensite plays a critical role in creating TWSME surfaces. DOI: 10.1557/mrc.2014.37 
A Engaged Learning of Materials Science and Engineering in the $21^{\text {st }}$ Century

\section{BIOMIATERIALS AND SOFT MATERIALS}

B Stretchable and Active Polymers and Composites for Electronics and Medicine

C Tough, Smart and Printable Hydrogel Materials

D Biological and Bioinspired Materials in Photonics and ElectronicsBiology, Chemistry and Physics

E Engineering and Application of Bioinspired Materials

F Biomaterials for Regenerative Engineering

G Plasma Processing and Diagnostics for Life Sciences

H Multifunctionality in Polymer-Based Materials, Gels and Interfaces

I Nanocellulose Materials and Beyond-

Nanoscience, Structures, Devices and Nanomanufacturing

$J$ Wetting and Soft Electrokinetics

K Materials Science, Technology and Devices for Cancer Modeling, Diagnosis and Treatment

L Nanofunctional Materials, Nanostructures and Nanodevices for Biomedical Applications

\section{NANOMIATERIALS AND SYNTHESIS}

M Micro- and Nanoscale Processing of Materials for Biomedical Devices

N Magnetic Nanomaterials for Biomedical and Energy Applications

0 Plasmonic Nanomaterials for Energy Conversion

P Synthesis and Applications of Nanowires and Hybrid 1D-0D/2D/3D Semiconductor Nanostructures

Q Nano Carbon Materials-1D to 3D

R Harsh Environment Sensing-Functional Nanomaterials and Nanocomposites, Materials for Associated Packaging and Electrical Components and Applications

\section{MECHANICAL BEHAVIOR AND FAILURE OF MATERIALS}

$S$ Mechanical Behavior at the Nanoscale

T Strength and Failure at the Micro- and NanoscaleFrom Fundamentals to Applications

U Microstructure Evolution and Mechanical Properties in Interface-Dominated Metallic Materials

V Gradient and Laminate Materials

W Materials under Extreme Environments (MuEE)

Y Shape Programmable Materials

\section{ELECTRONICS AND PHOTONICS}

Z Molecularly Ordered Organic and Polymer SemiconductorsFundamentals and Devices

AA Organic Semiconductors-Surface, Interface and Bulk Doping

BB Innovative Fabrication and Processing Methods for Organic and Hybrid Electronics

CC Organic Bioelectronics-

From Biosensing Platforms to Implantable Nanodevices

DD Diamond Electronics, Sensors and BiotechnologyFundamentals to Applications

EE Beyond Graphene-2D Materials and Their Applications

FF Integration of Functional Oxides with Semiconductors

GG Emerging Materials and Platforms for Optoelectronics

HH Optical Metamaterials-

From New Plasmonic Materials to Metasurface Devices

II Phonon Transport, Interactions and Manipulations

in Nanoscale Materials and Devices-Fundamentals and Applications

JJ Multiferroics and Magnetoelectrics

KK Materials and Technology for Non-Volatile Memories

\section{ENERGY AND SUSTAINABILITY}

LL Materials and Architectures for Safe and Low-Cost Electrochemical Energy Storage Technologies

MM Advances in Flexible Devices for Energy Conversion and Storage

NN Thin-Film and Nanostructure Solar Cell Materials and Devices for Next-Generation Photovoltaics

00 Nanomaterials-Based Solar Energy Conversion

PP Materials, Interfaces and Solid Electrolytes for High Energy Density Rechargeable Batteries

QQ Catalytic Materials for Energy

RR Wide-Bandgap Materials for Energy EfficiencyPower Electronics and Solid-State Lighting

SS Progress in Thermal Energy ConversionThermoelectric and Thermal Energy Storage Materials and Devices

\section{THEORY, CHARACTERIZATION AND MODELING}

TT Topology in Materials ScienceBiological and Functional Nanomaterials, Metrology and Modeling

UU Frontiers in Scanning Probe Microscopy

VV In Situ Study of Synthesis and Transformation of Materials

WW Modeling and Theory-Driven Design of Soft Materials

XX Architected Materials-Synthesis, Characterization, Modeling and Optimal Design

YY Advanced Atomistic Algorithms in Materials Science

ZZ Material Design and Discovery via Multiscale Computational Material Science AAA Big Data and Data Analytics for Materials Science

BBB Liquids and Glassy Soft Matter-Theoretical and Neutron Scattering Studies

CCC Integrating Experiments, Simulations and Machine Learning to Accelerate Materials Innovation

DDD Lighting the Path towards Non-Equilibrium Structure-Property Relationships in Complex Materials

$x \quad$ Frontiers of Material Research

\section{www.mrs.org/fall2015}

The MRS/E-MRS Bilateral Conference on Energy will be comprised of the energy-related symposia at the 2015 MRS Fall Meeting.

\section{Meeting Chairs}

T. John Balk University of Kentucky Ram Devanathan Pacific Northwest National Laboratory George G. Malliaras Ecole des Mines de St. Etienne Larry A. Nagahara National Cancer Institute Luisa Torsi University of Bari "A. Moro"

Don't Miss These Future MRS Meetings! 2016 MRS Spring Meeting \& Exhibit March 28 - April 1, 2016

Phoenix, Arizona

2016 MRS Fall Meeting \& Exhibit November 27 - December 2, 2016 Boston, Massachusetts 